\title{
Absolute-Structure Determination: Past, Present and Future
}

\author{
Howard D. Flack ${ }^{*}$
}

\begin{abstract}
Single-crystal X-ray crystallography is the major analytical technique in use today for absoluteconfiguration determination. The origins of absolute-structure determination, starting from Friedel's 1913 proof that the intensities of the opposites $h k l$ and $\bar{h} \bar{k} \bar{l}$ are identical, are traced. The important structural principles derived from the study of chiral, but pseudo-mirror symmetric, methyprylon are described. For the present time, the use of the average and difference intensities of the opposites $h k l$ and $\bar{h} \bar{k} \bar{l}$ are stressed. This leads to the use of Friedif, of $2 A D$ and selected $D$ plots, of $R_{\text {merge }}$ and the $D$-Patterson. The best techniques for absolute-structure determination in the future are described. Some advice to the scientific community concludes the paper.
\end{abstract}

Keywords: Absolute configuration · Absolute structure · Flack parameter · Resonant scattering · X-ray crystallography

\section{Introduction}

The unambiguous determination of the absolute structure of a single crystal, particularly in cases for which the absolute configuration of an enantiomerically-pure chiral molecule within the crystal is needed, is of importance not only for synthetic and natural-product chemists, who wish to fully characterize their products, but can be a critical step for the pharmaceutical industry, where opposite enantiomers of a drug can have quite different biological properties. In solid-state material science, the presence or absence of a centre of inversion or of twinning by inversion (i.e. the knowledge of absolute structure) can have important consequences for the physical properties of a material. Singlecrystal X-ray crystallography is the major analytical technique in use today for absolute-configuration determination.

\section{Past}

Historically, the determination of absolute structure started on the wrong foot with Friedel[1] in 1913 who, by a false argument using optics and crystal symmetry, managed to prove that the intensities of the Bragg reflections $h k l$ and $\bar{h} \bar{k} \bar{l}$ are always identical (Friedel's Law), regard-

${ }^{*}$ Correspondence: Dr. H. D. Flack

University of Geneva

Chimie minérale, analytique et appliquée

Geneva, Switzerland

E-mail: crystal@flack.ch less of the point group of the crystal. The effects of this unfortunate mistake are still felt today in the teaching of diffraction by crystals, as Friedel's Law is often presented and used as a fundamental principle whereas it is no more than a rough approximation, which often does not need to be invoked. In 1928, resonant scattering was predicted theoretically.[2] This phenomenon (sometimes called anomalous scattering or anomalous dispersion) is no more or no less than the natural response of a forced damped harmonic oscillator, so it is not even anomalous. In 1930, the first determination of absolute structure by $\mathrm{X}$-ray diffraction appeared as a by-product of a carefully contrived experiment ${ }^{[3]}$ to demonstrate the existence of resonant scattering. A non-centrosymmetric and achiral crystal of hexagonal $\mathrm{ZnS}$ (zincblende) was used in an experiment with Au $L$ radiation. The latter has a wavelength corresponding to the $K$ absorption edge of $\mathrm{Zn}$ which falls between the $\mathrm{Au} L_{\alpha 1}$ and $\mathrm{Au}$ $L_{\alpha 2}$ lines. The first chemical application of absolute-structure determination by X-ray diffraction was published ${ }^{4]}$ in 1951 . The absolute configuration of the $(2 R, 3 R)$ tartrate anion in its $\mathrm{Na} / \mathrm{Rb}$ salt was studied. Until 1990, routine absolute-structure determinations generally relied on comparing conventional $R$ factors for inversion-related structures, often in conjunction with Hamilton's $R$-factor ratio test. ${ }^{[5]}$ The modern developments of absolute-structure determination by least-squares refinement find their source in Rogers' (1981) paper. ${ }^{6]}$ Much of the resulting innovation centred on the Laboratory of Crystallography at the University of Geneva whose head was Professor Erwin Parthé. In an oft-quoted paper, Flack (1983),[7] it was discovered that every non-centrosymmetric crystal structure should be treated as a crystal twinned by inversion. In such a twin, the macroscopic crystal is composed of two inversion-related crystalline components whose lattices are perfectly aligned one with another. It is as though the crystals in a racemic conglomerate have been stuck together with a perfect alignment of their lattices. The Flack parameter is the basis of all modern absolute-structure determinations and its properties have been studied extensively in Geneva in various collaborations over thirty years. ${ }^{[8]}$ The Flack parameter is the molar fraction $x$ in the defining equation $C=(1-x) X+x(\bar{X})$, where $C$ represents an oriented two-domain-structure crystal twinned by inversion, consisting of an oriented domain structure $X$ and an oriented inverted domain structure $(\bar{X})$. In reciprocal space the Flack parameter $x$ is defined by the structure-amplitude equation $I(h k l, x)=(1-x)|F(h k l)|^{2}+x|F(\bar{h} \bar{k} \bar{l})|^{2}$. Various other studies have endeavoured to improve the precision of absolute-structure determination. [9]

The term absolute structure ${ }^{[10]}$ itself was coined in 1984. It is apposite to recall how absolute structure and absolute configuration are related one to another. Here are their current definitions:

Absolute configuration: The spatial arrangement of the atoms of a physically identified chiral molecular entity (or group) and its stereochemical description (e.g. $R$ or $S, P$ or $M, D$ or $L$ etc.).

Absolute structure: The spatial arrangement of the atoms of a physically identified noncentrosymmetric crystal and its description by way of unit-cell dimensions, space group and representative coordinates of all atoms.

Absolute structure is a crystallographer's term and applies to noncentrosym- 
metric crystal structures. Absolute configuration is a chemist's term and refers to chiral molecules. Note particularly that both the entity under consideration, viz. crystal structure versus molecule, and the symmetry restrictions, viz. noncentrosymmetric versus lack of mirrors, centres of symmetry and roto-inversions, are different. Both terms concern the complete specification of the spatial arrangement of atoms with respect to inversion.

In the domain of organic chemical crystallography, there is a scientific publication in Helvetica Chimica Acta emanating from the Swiss pharmaceutical industry in Basel, which describes some important and surprising structural principles. ${ }^{[11]}$ On crystals of 3-diethyl-5-methylpiperidine-2,4-dione (methyprylon), thermochemical, DSC, contact-method $^{[12]}$ and crystallographic ${ }^{[11]}$ measurements have been made. The molecule presents approximate mirror symmetry although it is chiral. The space-filling diagrams, displayed in Fig. 1, show that the shapes of the two opposite enantiomers are very similar indeed. It comes as no surprise that the opposite enantiomers form a continuous range of solid solutions (mixed crystals) from enantiomerically-pure $S$-methyprylon to enantiomerically-pure $R$-methyprylon. A further significant observation is that the enantiomerically-pure samples are dimorphic. Both of the solid phases crystallize in the space-group type $P 22_{1} 2_{1}$ (modification I: $a=13.12, b$ $=11.69, c=6.83 \AA$; modification II: $a=$ $12.35, b=12.30, c=6.83 \AA$ ) with essentially the same cell parameter $c$ whilst $a$ and $b$ vary linearly as a function of composition as determined by the optical rotation of a crop of crystals as seen in Fig. 2. The dimorphism seems inevitable in such a case. Consider the crystal structure of the enantiomerically-pure $S$-methyprylon in the solid phase I. Let us call this the $L$ crystal structure or to be precise, $L$ decorated by $S$. As this crystal structure is formed of enantiomerically-pure molecules it must be chiral. The enantiomorph of the $L$ decorated by $S$ crystal structure is the $D$ crystal structure formed of pure $R$-methyprylon molecules i.e. the $D$ decorated by $R$ crystal structure. Now in the $L$ decorated by $S$ crystal structure consider gradually replacing the $S$-methyprylon by $R$-methyprylon molecules. As end point of this replacement, we will obtain the $L$ decorated by $R$ crystal structure composed entirely of $R$-methyprylon molecules. Clearly this new structure, $L$ decorated by $R$, is not the enantiomorph of the starting structure, $L$ decorated by $S$. The enantiomorph of $L$ decorated by $R$ is $D$ decorated by $S$ and the enantiomorph of $L$ decorated by $S$ is $D$ decorated by $R$. Clearly for the enantiomerically pure $S$-methyprylon molecules there are two crystal structures,

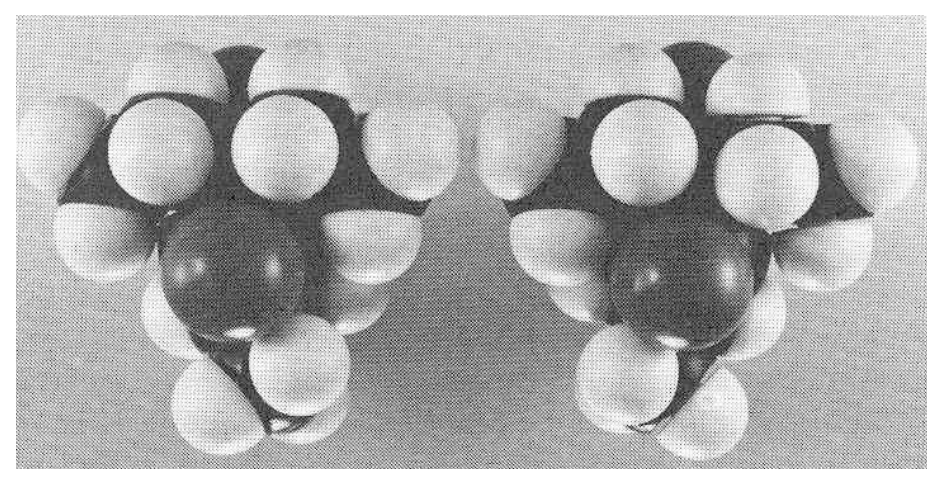

Fig. 1. Space filling representations of the opposite enantiomers of methyprylon. The differences between the two are slight. Reproduced with permission from $\mathrm{W}$. E. Oberhänsli, Helv. Chim. Acta 1982 65, 924-933. Copyright John Wiley \& Sons, 1982.

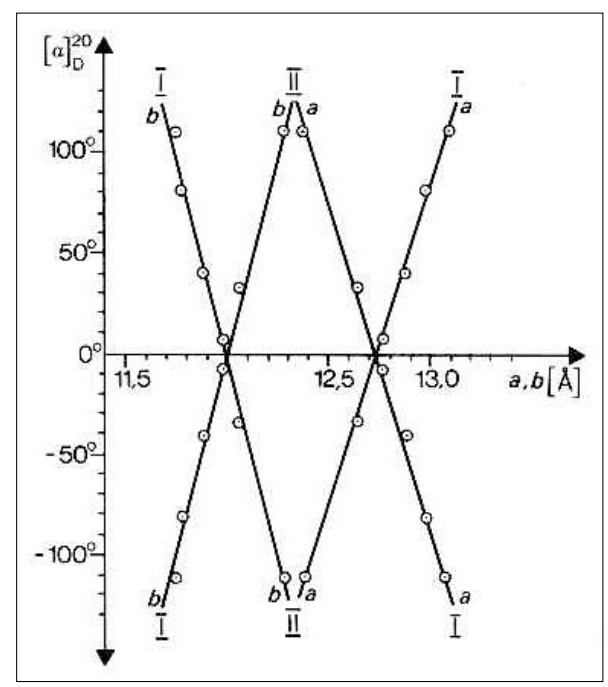

Fig. 2. Variation of $a$ and $b$ cell dimensions of the dimorphs of methyprylon as a function of composition as determined by the optical rotation of a crop of crystals. Reproduced with permission from W. E. Oberhänsli, Helv. Chim. Acta 1982 65, 924-933. Copyright John Wiley \& Sons, 1982.

$L$ decorated by $S$ and $D$ decorated by $S$, each with its own enantiomorph formed of enantiomerically-pure $R$-methyprylon molecules.

\section{Present}

It is commonly admitted that the most important remaining problem in absolute-structure determination is the insufficient precision of the Flack parameter of light-atom structures as determined by standard least-squares techniques. Without doubt, the requirements for absolute-configuration determination both of the pharmaceutical industry and of synthetic organic chemists are a major driving force behind any development. For example, on a study of $\sim 100$ light-atom crystal structures, ${ }^{[13]}$ it was shown that the root-meansquare deviation from zero of the Flack parameters was notably smaller than the standard uncertainties on the individual determinations. This was taken to imply that standard least-squares refinement of the Flack parameter was producing stand- ard uncertainties on this parameter that are systematically overestimated (i.e. too large). Various techniques ${ }^{[9]}$ have been devised to improve the precision of absolute-structure determination. These rely on advanced statistical analyses and in many cases result in absolute-structure determinations of increased precision. A consequence of the light-atom syndrome is that there have been few studies devoted to the wider field of the application of resonant scattering in small-molecule crystallography. Nevertheless, we present in outline, those studies available, in the hope that they will stimulate further development in absolute-structure determination and in small-molecule crystallography in general. Fuller details can be found in the publications. ${ }^{\left[{ }^{[8]}\right.}$ In much of the following, great use is made of the average $(A)$ and difference (D) of Friedel opposites, $h k l$ and $\bar{h} \bar{k} \bar{l}$, defined as:

$$
\begin{aligned}
& A(h k l)=1 / 2\left[|F(h k l)|^{2}+|F(\bar{h} \bar{k} \bar{l})|^{2}\right] \\
& D(h k l)=|F(h k l)|^{2}-|F(\bar{h} \bar{k} \bar{l})|^{2} .
\end{aligned}
$$

\subsection{Friedif}

The need was felt for a quantitative estimate of the potential resonant-scattering signal to be found in diffraction measurements from non-centrosymmetric crystal structures. A natural choice for such an estimator was the root-mean-square value of $D$ divided by the mean value of $A$, i.e. $\left\langle D^{2}\right\rangle^{1 / 2} /<A>$. Employing the techniques of intensity statistics to a random structure in space group $P 1$ with many Bragg reflections, it was found that a value, now called Friedif $_{\text {stat }}$, could be calculated from the presumed chemical composition of the crystal and the wavelength of the X-radiation. ${ }^{[14]}$ Light-atom structures measured with $\mathrm{MoK} \alpha$ radiation give Friedif ${ }_{\text {stat }}$ values less than 10. Heavy-atom structures can give values over 1000 . Friedif ${ }_{\text {model }}$ could be calculated from the model (calculated) structure-factor amplitudes available for $\sim 100$ published crystal structures. These rely on a refined model of each crystal structure and they covered a large number of different space groups and chemical compositions. It was found that Friedif is a very robust estimator of Friedif ${ }^{\text {stat }}$ Moreover Friedif ${ }_{\text {obs }}$ can be calculated from 
the observed structure-factor amplitudes. Its calculation is somewhat tricky as one must take proper account of the average $\sin \theta / \lambda$ dependence of the $A$ and $D$ values, which are different one from another. Comparison of Friedif ${ }_{\mathrm{obs}}$ with Friedif leads to a priori indications of whether the crystal is centrosymmetric or not, whether it is twinned by inversion or not and whether the resonant-scattering signal has been preserved in the $D$ values and not swamped out by random uncertainties and systematic error.[14]

\subsection{AD and Selected D Plots}

Until recently the evaluation of absolute-structure determination had been based entirely on the values of parameters derived from the least squares, particularly the Flack parameter and its standard uncertainty. Scant attention was paid to the fit of the model to the observed diffraction intensities. Dr. David Watkin of the University of Oxford in England found a very revealing and simple method to display the fit. ${ }^{[15]}$ It had been customary to display the fit of the data after least-squares refinement by plotting $\left|F_{\text {obs }}(h k l)\right|^{2}$ against $\left|F_{\text {calc }}(h k l)\right|^{2}$. On such a plot, a good fit is displayed by the data points following a straight line of slope 1 passing through the origin. A false scale factor, untreated extinction and outliers may be readily identified on such a plot. The fit can be seen from the spread of the points around the ideal line. The Watkin hybrid ${ }^{[15]}$ is to plot $A_{\text {obs }}$ against $A_{\text {model }}$ and $D_{\text {obs }}$ against $D_{\text {model }}$ as seen for compounds gz3201 and fa3274 in Fig. 3. The $A_{\text {obs }}, A_{\text {mode }}$ plot has the same interpretation as the $|F|^{2}$ plot. However the $D_{\text {obs }}, D_{\text {model }}$ plot held un- suspected surprises. About 33\% of published non-centrosymmetric crystal structures (those in Acta Cryst. C in 2011 and 2012) showed plots of the expected form as seen in Fig. 3(a) for gz3201. It is suspected for these crystal-structure determinations that the standard uncertainty of the Flack parameter obtained by conventional leastsquares refinement is overestimated (i.e. too large). Another $33 \%$ of these published non-centrosymmetric crystal structures showed $D_{\text {obs }}, D_{\text {model }}$ plots of a different form. The data points were all spread around the $D_{\text {obs }}$ axis near to $D_{\text {model }}=0$ and the range of $\left|D_{\text {obs }}\right|$ values was far greater than the range of $\left|D_{\text {model }}\right|$, as seen in Fig. 3(b) for fa3274. Such plots are interpreted as indicating that the resonant-scattering signal is hidden by the random uncertainties and systematic errors in the $D_{\text {obs }}$. It is suspected for these crystal-structure determinations that the standard uncertainty of the Flack parameter obtained by conventional leastsquares refinement is underestimated (i.e. too small). The remaining $33 \%$ of these crystal-structure determinations had intermediate behaviour.

In a further development of these plots, it has been found useful to plot $D_{\text {obs }}$ against $D_{\text {single }} . D_{\text {single }}$ is the value of $D_{\text {model }}$ for a single crystal untwinned by inversion. On such a plot it is possible to reject outliers according to various criteria. One criterion is to reject all $D_{\text {obs }}, D_{\text {single }}$ data points which have $\left|D_{\text {obs }}\right|>2 \mid D_{\text {single }}^{\text {singax }}$. Another useful criterion is to reject reflections with a low value of $\left|A_{\text {obs }}\right|$. Such a plot can be used not only as a data evaluation technique but also to determine the value of the Flack parameter and its standard uncertainty. A slope of 1, corresponds to a Flack parameter of zero, and a slope of zero to a Flack parameter of 0.5 .

\section{3 $\boldsymbol{R}_{\text {merg }}$}

At the outset of a crystal-structure determination it is customary to make tests of the possible symmetry-equivalence of the intensities of sets of Bragg reflections in order to determine the approximate centrosymmetric point group of the intensities in the diffraction pattern. This is known as the Laue group of the crystal. The statistic $R_{\text {merge }}$ is used for this purpose. A few tests have been undertaken on selected crystals to see whether it is possible to go further

Table 1. $R$ merging values [\%] for the 589 sets of $\mathrm{mmm}$-general reflections which have all eight measurements in the set for $\mathrm{K} \mathrm{H}(2 R, 3 R)$ tartrate. Reproduced with permission from S. Parsons, P. Pattison, H. D. Flack, Acta Cryst. 2012 A68, 736-749. Copyright International Union of Crystallography.

\begin{tabular}{|l|l|l|l|l|l|}
\hline & $m m m$ & $2 m m$ & $m 2 m$ & $m m 2$ & 222 \\
\hline$R_{\mathrm{IF|}}^{2}[\%]$ & 2.42 & 2.31 & 2.29 & 2.31 & 1.80 \\
\hline$R_{\mathrm{A}}[\%]$ & 1.30 & 1.30 & 1.30 & 1.30 & 1.30 \\
\hline$R_{\mathrm{D}}[\%]$ & 100.0 & 254.4 & 235.7 & 258.1 & 82.9 \\
\hline
\end{tabular}

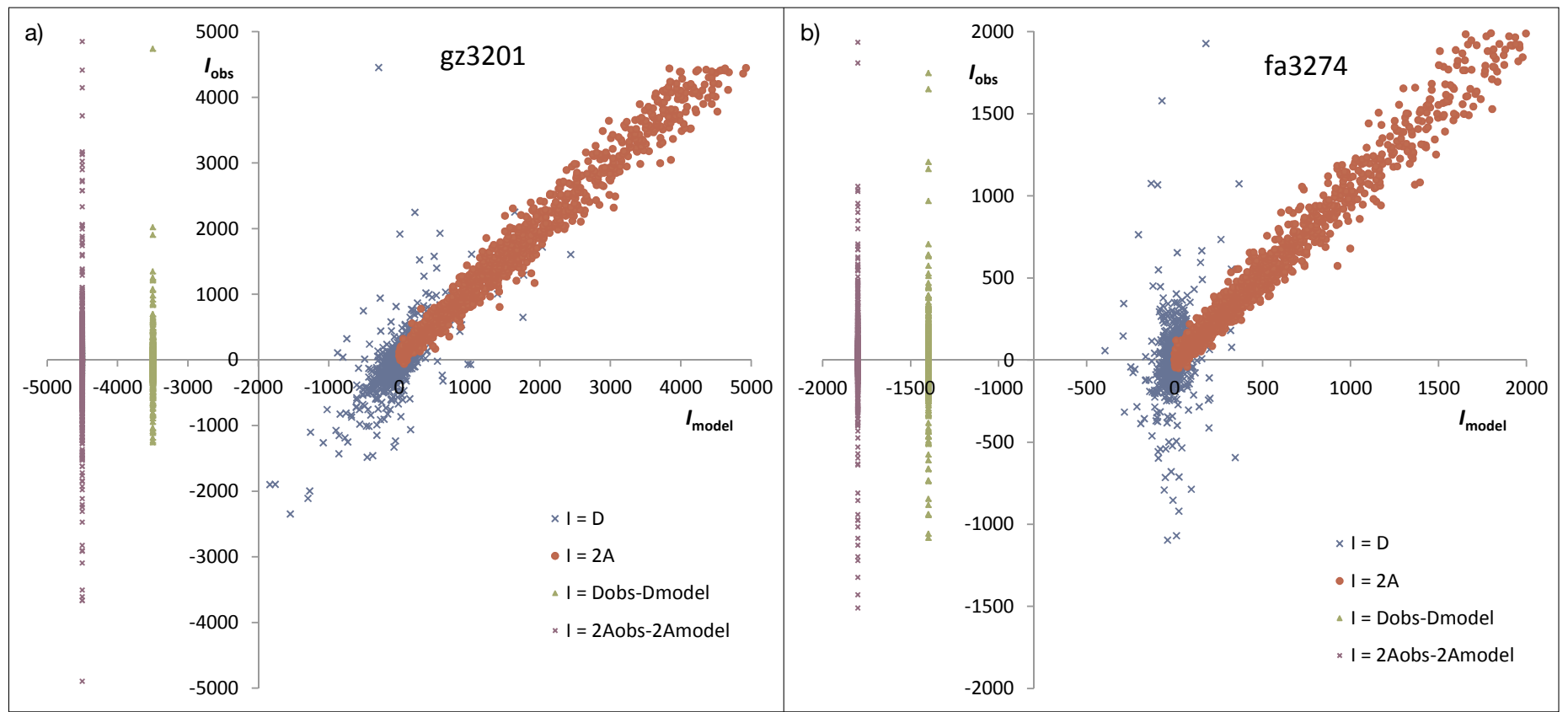

Fig. 3. $2 A D$ plots for gz3201 in (a) and fa3274 in (b). Both gz3201 and fa3274 have Friedif ${ }_{\text {stat }} \sim 470$. gz3201 shows a good fit of $D_{\text {obs }}$ to $D_{\text {model }}$, whereas for fa3274 the $D_{\text {obs }}$ are dominated by random uncertainties and systematic errors. Reproduced with permission from H. D. Flack, Acta Cryst. 2013 C69, 803-807. Copyright International Union of Crystallography. 
in such an analysis and determine not only the Laue group but also the point group of the crystal. The results are very encouraging. As can be seen in Table 1, for $\mathrm{K} \mathrm{H}(2 R$, $3 R$ ) tartrate, the point group 222 is clearly superior to $2 \mathrm{~mm}, \mathrm{~m} 2 \mathrm{~m}, \mathrm{~mm} 2$ and $\mathrm{mmm}^{[16]}$ when using $R_{\mathrm{D}}$ as the appropriate statistic. For an unequivocal result, one needs all of the Laue-class symmetry-equivalent reflections to have been measured for each general $h k l$. However these tests have shown up shortcomings in the data acquisition. Data-sets which seemed at first sight to be highly redundant, nevertheless contained a considerable number of sets of reflections lacking some of the Laue-class symmetry-equivalent reflections.

\subsection{D-Patterson}

The Friedel-difference intensities can be exploited by way of the antisymmetric sine Patterson function. It is calculated using the Friedel-difference intensities $(D)$. The $D$-Patterson shows, as usual, peaks at the positions of interatomic vectors but those between atoms of the same chemical element do not appear. This makes the $D$-Patterson map less inundated with peaks compared to the usual $A$-Patterson. It has been shown that the $D$-Patterson is useful in structure evaluation to identify displaced or incorrectly assigned atoms. Also in the intermetallic compound $\mathrm{TiGePt}$, it was critical in deciding whether the crystal structure was centrosymmetric or not.[17]

\section{Future}

It is most appropriate for an article of this type that a clear indication be given of the manner in which the particular problems of the least-squares refinement of non-centrosymmetric crystal structures could be best treated. These procedures are designed to lead to a reliable value of the Flack parameter with as low and realistic a standard uncertainty as possible. Of course, the current author accepts full responsibility for the opinions expressed here. There are two situations which need to be treated separately. In the first case, the determination of absolute structure, and most probably the absolute configuration of the constituent chiral molecules of the crystal, is a prime objective of the crystal-structure determination alongside the determination of molecular geometry. In the second case, the only objective is the determination of molecular geometry.

The procedures described below make the best use of results coming from recent publications, ${ }^{[18,19]}$ and provide not only numerical values to describe the absolute structure of a crystal but simultaneously display the fit obtained between the observed and model diffraction intensities. The pro- cedures are not yet being used routinely because the generally available software packages do not offer the necessary options.

\subsection{Procedure absolute-structure determination}

(i) The reflection data-set is separated into three disjoint classes consisting of centric reflections (class $c$ ), pairs of Friedel opposites $(h k l$ and $\bar{h} \bar{k} \bar{l})$ of acentric reflections (class $a p$ ) and unpaired acentric reflections (class $a u$ ). If the data-set contains an unacceptably large proportion of unpaired acentric reflections, the diffraction data should be recollected with a revised collection strategy. The data in class $a u$ are omitted from further analysis.

(ii) The intensities of the pairs of acentric reflections (class $a p$ ), are transformed into averages, $A_{\mathrm{obs}}(h k l)$, and differences, $D_{\text {obs }}(h k l)$.

(iii) Least-squares refinement of the structural parameters is undertaken using as data the centric reflections (class $c$ ) and the average intensities of the paired Friedel opposites $\left[A_{\text {obs }}(h k l)\right]$. This refinement corresponds to a crystal twinned by inversion in a proportion of 50:50, so a value of the Flack parameter fixed at 0.5 should be used. The resulting atomic parameters are unbiased by the effects of resonant scattering and inversion twinning.

(iv) Using the atomic parameters obtained from stage (iii) with a Flack parameter set to 0.0 , structure-factor amplitudes are calculated for the pairs of Friedel opposites in class $a p$. This leads to model values $D_{\text {single }}(h k l)$ corresponding to a single crystal untwinned by inversion.

(v) From a plot of $D_{\text {bs }}(h k l)$ against $D_{\text {single }}(h k l)$ one has a powerful tool for validating the absolute-structure determination and obtaining a value of the Flack parameter with its standard uncertainty from a least-squares fit to a straight line passing through the origin. Critical to the success of this fit is the choice of a suitable weighting scheme and the elimination of outliers.

The transformation of the data described in (ii) yields one set of observations [the centric reflections and $A_{\text {obs }}(h k l)$ ] which is sensitive to the structure but independent of the Flack parameter, and another, $D_{\text {obs }}(h k l)$, which is sensitive to the Flack parameter, but highly insensitive to the atomic parameters. The agreement between $A_{\text {obs }}(h k l)$ and $A_{\text {model }}(h k l)$ is usually much better than between $D_{\text {obs }}(h k l)$ and $D_{\text {model }}(h k l) .{ }^{[18]}$ One advantage of the transformation of the $|F(h k l)|^{2}$ data into $A(h k l)$ and $D(h k l)$ is that different schemes for the selection of outliers and weights can be applied to each. Use of the transformed data also removes correlation between the Flack parameter and the other structural parameters.

\subsection{Procedure no interest in absolute structure}

(i) Do not average Friedel opposites and do not classify the reflections into the centric (c), paired acentric ( $a p)$ and unpaired acentric $(a u)$ classes.

(ii) Refine the Flack parameter by full-matrix least squares working on $|F(h k l)|^{2}$.

The following points have been taken into account in designing this procedure.

(a) The experimenter cannot be required to make measurements in which all Friedel opposites are measured. Consequently there may be a considerable number of unpaired acentric $(a u)$ reflections for which $A_{\text {obs }}(h k l)$ and $D_{\text {obs }}(h k l)$ cannot be calculated.

(b) Some experiments yield reflection data which contain a clear resonant-scattering signal in the $D_{\text {obs }}(h k l)$ even if Friedif is low [i.e. a small resonant-scattering contribution to $D(h k l)$ ] whereas for some others, the resonant-scattering signal may not be discernible in the $D_{\text {obs }}(h k l)$ even for large Friedif $f_{\text {stat }}$ values.

(c) It is inappropriate to require that Friedel opposites be averaged as the data set inevitably contains both pairs of Friedel opposites of acentric reflections and unpaired acentric reflections. On averaging, the former provide a subset of the data corresponding to a Flack parameter of 0.5 and the latter a disjoint subset corresponding to the Flack parameter of the crystal.

\section{Concluding Remarks}

Absolute-structure determination is everybody's problem. It is definitely not reserved for some old tired experts to fill up their time after retirement. Synthetic chemists need to pester their structure analysts (service crystallographers) to know whether the most appropriate modern techniques such as those described in sections 3 and 4 have been used in the study of their crystals. The structure analyst has the perfect right, even obligation, to chase after software providers and instrument manufacturers to exalt them to provide these modern high-performance techniques and material. Authors, editors and referees of journal articles have collective responsibility one to another. The best techniques must be used, described and evaluated. And, last but not least, funding agencies and industrial sponsors need to provide generous support to undertake the necessary studies.

Received: November 7, 2013

[1] G. Friedel, C. R. Acad. Sci. Paris 1913, 157, 1533.

[2] I. Waller, Z. Phys. 1928, 51, 213. 
[3] D. Coster, K. S. Knol, J. Prins, Z. Phys. 1930, 63, 345 .

[4] a) J. M. Bijvoet, A. F. Peerdeman, A. J. Van Bommel, Nature 1951, 168, 271; b) A. F. Peerdeman, A. J. Van Bommel, J. M. Bijvoet, Proc. K. Ned. Akad. Wet. Ser. B 1951, 54, 16.

[5] W. C. Hamilton, Acta Cryst. 1965, 18, 502.

[6] D. Rogers, Acta Cryst. 1981, A37, 734.

[7] H. D. Flack, Acta Cryst. 1983, A39, 876.

[8] a) G. Bernardinelli, A. Dunand, H. D. Flack, K. Yvon, Acta Cryst. 1984, C40, 1911; b) G. Bernardinelli, H. D. Flack, Acta Cryst. 1985, A41, 500; c) G. Bernardinelli, H. D. Flack, Acta Cryst. 1987, A43, 75; d) H. D. Flack, D. Schwarzenbach, Acta Cryst. 1988, A44, 499; e) H. D. Flack, G. Bernardinelli, Acta Cryst. 1999, A55, 908; f) H. D. Flack, G. Bernardinelli, $J$. Appl. Cryst. 2000, 33, 1143; g) H. D. Flack, Helv. Chim. Acta 2003, 86, 905; h) M. Hostettler, H. D. Flack, Acta Cryst. 2003, B59, 537; i) H. D. Flack, G. Bernardinelli, Cryst. Engin. 2003, 6, 213; j) H. D. Flack, G. Bernardinelli, Inorg. Chim. Acta 2006, 359, 383; k) H. D. Flack, G. Bernardinelli, D. A. Clemente, A. Linden, A. L. Spek, Acta Cryst. 2006, B62, 695; 1) H. D. Flack, U. Shmueli, Acta Cryst. 2007, A63, 257; m) J.-P. Djukic, A. Hijazi, H. D. Flack, G. Bernardinelli, Chem. Soc. Rev. 2008, 37, 406; n) H. D. Flack, G. Bernardinelli, Chirality 2008, 20,681; o) U. Shmueli, M. Schiltz, H. D. Flack,
Acta Cryst. 2008, A64, 476; p) H. D. Flack; G. Bernardinelli, Acta Cryst. 2008, A64, 484; q) H. D. Flack, Acta Chim. Slovenica 2008, 55, 689; r) U. Shmueli, H. D. Flack, Acta Cryst. 2009, A65, 322; s) H. D. Flack, Acta Cryst. 2009, A65, 371 ; t) U. Shmueli, H. D. Flack, Acta Cryst. 2010, A66, 669; u) H. D. Flack, M. Sadki, A. L. Thompson, D. J. Watkin, Acta Cryst. 2011, A67, 21; v) S. Parsons, P. Pattison, H. D. Flack, Acta Cryst. 2012, A68, 736; w) S. Parsons, H. D. Flack, T. Wagner, Acta Cryst. 2013, B69, 249; x) H. D. Flack, Acta Cryst. 2013, C69, 803 ; y) S.-V. Ackerbauer, H. Borrmann, H.B. Bürgi, H. D. Flack, Y. Grin, A. Linden, L. Palatinus, W. B. Schweizer, R. Warshamanage, M. Wörle, Acta Cryst. 2013, B69, 457.

[9] a) R. W. W. Hooft, L. H. Straver, A. L. Spek, J. Appl. Cryst. 2008, 41, 96; b) R. W. W. Hooft, L. H. Straver, A. L. Spek, J. Appl. Cryst. 2010, 43, 665 ; c) Y. Le Page, E. J. Gabe, G. J. Gainsford, J. Appl. Cryst. 1990, 23, 406; d) S. Parsons, H. D. Flack Acta Cryst. 2004, A60, s61; e) S. Parsons, Acta Cryst. 2011, A67, C191; f) S. Parsons, Acta Cryst. 2012, A68, s4.

[10] a) P. G. Jones, Acta Cryst. 1984, A40, 660; b) H. D. Flack, G. Bernardinelli, Acta Cryst. 1999, A55, 908 .

[11] W. E. Oberhänsli, Helv. Chim. Acta 1982, 65, 924.
[12] a) M. Kuhnert-Brandstätter, K. Schleich, K. Vogler, Monatsh. Chem. 1970, 101, 1817; b) K. Vogler, M. Kofler, Helv. Chim. Acta 1956, 39 , 1387.

[13] A. L. Thompson, D. J. Watkin, J. Appl. Cryst. 2011, 44, 1017.

[14] a) H. D. Flack, G. Bernardinelli, Acta Cryst. 2008, A64, 484; b) H. D. Flack, Acta Chim. Slovenica 2008, 55, 689; c) H. D. Flack, M. Sadki, A. L. Thompson, D. J. Watkin, Acta Cryst. 2011, A67, 21; d) S. Parsons, P. Pattison, H. D. Flack, Acta Cryst. 2012, A68, 736; e) H. D. Flack, Acta Cryst. 2013, C69, 803.

[15] a) H. D. Flack, M. Sadki, A. L. Thompson, D. J. Watkin, Acta Cryst. 2011, A67, 21; b) S. Parsons, P. Pattison, H. D. Flack, Acta Cryst. 2012, A68, 736; c) H. D. Flack, Acta Cryst. 2013, C69, 803.

[16] S. Parsons, P. Pattison, H. D. Flack, Acta Cryst. 2012, A68, 736.

[17] a) H. D. Flack, M. Sadki, A. L. Thompson, D. J. Watkin, Acta Cryst. 2011, A67, 21; b) S.-V. Ackerbauer, H. Borrmann, H.-B. Bürgi, H. D. Flack, Y. Grin, A. Linden, L. Palatinus, W. B. Schweizer, R. Warshamanage, M. Wörle, Acta Cryst. 2013, B69, 457.

[18] a) H. D. Flack, M. Sadki, A. L. Thompson, D. J. Watkin, Acta Cryst. 2011, A67, 21; b) S. Parsons, P. Pattison, H. D. Flack, Acta Cryst. 2012, A68, 736.

[19] a) S. Parsons, H. D. Flack, T. Wagner, Acta Cryst. 2013, B69, 249; b) H. D. Flack, Acta Cryst. 2013, C69, 803; c) A. L. Spek, J. Appl. Cryst. 2003, 36, 7. 